\title{
Фотолюминесцентные исследования легированных кремнием эпитаксиальных пленок GaAs, выращенных на подложках GaAs с ориентациями (100) и (111)A при пониженных температурах
}

\author{
(С) Г.Б. Галиев, Е.А. Климов, А.Н. Клочков, С.С. Пушкарев, П.П. Мальцев
}

Институт сверхвысокочастотной полупроводниковой электроники Российской академии наук, 117105 Москва, Россия

E-mail: galiev_galib@mail.ru

(Получена 23 марта 2017 г. Принята к печати 5 апреля 2017 г.)

Исследованы электрофизические свойства и особенности фотолюминесценции однородно легированных атомами $\mathrm{Si}$ слоев $\mathrm{GaAs}$, выращенных методом молекулярно-лучевой эпитаксии на подложках $\mathrm{GaAs} \mathrm{c}$ кристаллографической ориентацией поверхности (100) и (111)А. Исследуемые образцы выращивались при одинаковом давлении $\mathrm{As}_{4}$ в интервале температур роста от 350 до $510^{\circ} \mathrm{C}$. Образцы на подложках $\mathrm{GaAs}(100)$ имеют $n$-тип проводимости во всем указанном интервале температур роста, а образцы на подложках $\mathrm{GaAs}$ (111)А имеют $p$-тип проводимости в интервале температур роста $430-510^{\circ} \mathrm{C}$. Спектры фотолюминесценции образцов содержат краевую и примесную полосы. Краевая фотолюминесценция соответствует фотолюминесценции вырожденного GaAs c $n$ - и $p$-типом проводимости. Примесную фотолюминесценцию образцов на подложках $\mathrm{GaAs}(100)$ в диапазоне $1.30-1.45$ эВ мы приписываем дефектам $V_{\mathrm{As}}$ и комплексам дефектов $\mathrm{Si}_{\mathrm{As}}-V_{\mathrm{As}}$, концентрация которых изменяется с температурой роста образцов. Трансформация спектров примесной фотолюминесценции образцов на подложках GaAs (111)A интерпретирована как обусловленная изменением концентрации дефектов $V_{\mathrm{As}}$ и $V_{\mathrm{Ga}}$ при изменении температуры роста образцов.

DOI: 10.21883/FTP.2018.03.45628.8589

\section{1. Введение}

Интерес к пленкам соединений $\mathrm{A}^{\mathrm{III}} \mathrm{B}^{\mathrm{V}}$, выращенным методом молекулярно-лучевой эпитаксии (МЛЭ) в низкотемпературном режиме, вызван возможностью использования их в устройствах терагерцевого диапазона [1]. Фотопроводящие терагерцевые антенны должны обладать ультракоротким временем жизни фотовозбужденных носителей, большим удельным сопротивлением и высокой подвижностью носителей заряда [2]. Удовлетворяющим таким требованиям материалом является GaAs, полученный при низких температурах эпитаксиального роста (low-temperature GaAs, LT-GaAs) [2,3]. Указанные специфические свойства LT-GaAs обусловлены наличием избыточных атомов As в объеме эпитаксиальной пленки, которые приводят к образованию следующих точечных дефектов: атом мышьяка в узле атома галлия $\left(\mathrm{As}_{\mathrm{Ga}}\right)$, межузельный атом мышьяка $\left(\mathrm{As}_{i}\right)$, вакансия атома галлия $\left(V_{\mathrm{Ga}}\right)$. Считается, что за субпикосекундное время жизни фотовозбужденных носителей заряда и большое удельное сопротивление в LT-GaAs ответственны ионизированные точечные дефекты $\mathrm{As}_{\mathrm{Ga}}^{+}$, которые являются глубокими донорами [4,5]. Применяется легирование LT-GaAs атомами Be, которые создают в GaAs мелкие акцепторные уровни, увеличивают концентрацию ионизированных дефектов $\mathrm{As}_{\mathrm{Ga}}^{+}$и тем самым уменьшают время захвата электронов [4]. Однако использование источника бериллия в установке МЛЭ ограничивается его высокой токсичностью и повышением концентрации акцепторной фоновой примеси во всех гетероструктурах, выращиваемых в дальнейшем в дан- ной установке. Поэтому поиск подходящей акцепторной примеси для LT-GaAs является актуальной задачей.

B фотопроводящих антеннах используются как нелегированные $[6,7]$, так и легированные атомами Ве эпитаксиальные пленки LT-GaAs $[4,5,8,9]$. Электрофизические и структурные характеристики LT-GaAs можно оптимизировать, варьируя следующие технологические параметры: температуру роста $T_{g}$, соотношение потоков мышьяка и галлия $\gamma=P_{(\mathrm{As})} / P_{\mathrm{Ga}}$, температуру и время послеростового отжига. При использовании легированных бериллием пленок LT-GaAs необходимо оптимизировать также и концентрацию атомов Ве в пленке [5].

Как известно, атомы $\mathrm{Si}$, являясь легирующей примесью, в GaAs проявляют амфотерные свойства. Особенно ярко амфотерное поведение $\mathrm{Si}$ проявляется при использовании для эпитаксиального роста подложек GaAs с кристаллографической ориентацией поверхности $(n 11) \mathrm{A}$, где $n=1,2,3 \ldots$ На таких подложках можно вырастить эпитаксиальные пленки GaAs, легированные $\mathrm{Si}$ (GaAs: $\mathrm{Si}$ ), как с $n$-, так и с $p$-типом проводимости, а также компенсированные (непроводящие) пленки [10-13]. Основные экспериментальные данные получены для эпитаксиальных пленок GaAs, выращенных при стандартных температурах роста $\left(450-550^{\circ} \mathrm{C}\right)$. В работе [14] представлены результаты исследований электрофизических параметров легированных кремнием эпитаксиальных пленок GaAs на подложках $\mathrm{GaAs}$ (311)A, выращенных при фиксированном значении $\gamma$ и при более низких значениях $T_{g}$ : от $\sim 600$ до $380^{\circ} \mathrm{C}$.

Спектроскопия фотолюминесценции (ФЛ) может успешно применяться для диагностики точечных дефектов $V_{\mathrm{As}}, V_{\mathrm{Ga}}, \mathrm{As}_{\mathrm{Ga}}, \mathrm{Ga}_{\mathrm{As}}$ и комплексов $V_{\mathrm{As}}-\mathrm{Si}_{\mathrm{As}}$, 
$\mathrm{Si}_{\mathrm{Ga}}-V_{\mathrm{Ga}}$ в $\mathrm{GaAs}$ [15]. Концентрации $\mathrm{As}_{\mathrm{Ga}}$ и $\mathrm{Ga}_{\mathrm{As}}$ могут достигать $10^{20} \mathrm{~cm}^{-3}$, а концентрации $V_{\mathrm{Ga}}$ и $V_{\mathrm{As}}-$ $10^{18} \mathrm{~cm}^{-3}$, и их можно изменять в широких пределах в зависимости от условий роста [16-18]. Такие дефекты влияют на электрофизические и оптические свойства $\mathrm{GaAs}$, поскольку принимают участие в компенсации легирующих примесей и выступают в качестве ловушек, рекомбинационных и рассеивающих центров. Именно такие дефекты, как уже отмечалось ранее, определяют основные свойства фотопроводящих антенн на основе LT-GaAs. Подробный анализ спектров ФЛ эпитаксиальных пленок GaAs, легированных $\mathrm{Si}$, на подложках GaAs (100) и (n11)A приведен в работах [12,13,19]. Данные исследования также относятся к структурам, выращенным при стандартных температурах роста.

Таким образом, в опубликованной литературе недостаточно исследований электрофизических и оптических свойств легированных кремнием пленок GaAs, выращенных при низкотемпературных условиях на подложках с ориентациями $(n 11) \mathrm{A}$. В частности, недостаточно исследована возможность получения $p$-типа проводимости в слоях GaAs : Si на подложках с ориентацией (n11)A при приближении к низкотемпературным условиям эпитаксиального роста. Целью настоящей работы является исследование типа проводимости и спектров ФЛ однородно легированных кремнием эпитаксиальных пленок GaAs, выращенных на подложках GaAs с ориентациями (100) и (111)А при пониженных температурах роста.

\section{2. Образцы и методы исследования}

Исследуемые образцы были выращены методом МЛЭ на полуизолирующих подложках GaAs с кристаллографическими ориентациями поверхности (100) и (111)A. Будем обозначать образцы, выращенные на подложках GaAs (100), как (номер образца)-0, а на подложках GaAs (111)A - как (номер образца)-1. Для достижения максимальной идентичности технологических условий (температуры роста $T_{g}$ и соотношения давлений тетрамеров мышьяка и атомов галлия $\left.\gamma=P_{\mathrm{As}_{4}} / P_{\mathrm{Ga}}\right)$ в одном процессе на держатель образца монтировались две половины подложек $\mathrm{GaAs}$ с разными ориентациями. В каждом процессе после стандартной процедуры предростового отжига образца выращивался нелегированный буферный слой GaAs толщиной 120 нм при стандартной температуре роста $520^{\circ} \mathrm{C}$. После этого выращивался легированный кремнием слой $\mathrm{GaAs}$ толщиной 864 нм. Температура кремниевой ячейки при выращивании легированного слоя составляла $1120^{\circ} \mathrm{C}$, что соответствовало в наших условиях значению концентрации электронов $4 \cdot 10^{18} \mathrm{~cm}^{-3}$ при высокотемпературном росте пленки $\mathrm{GaAs}$ : Si на подложке GaAs (100). Легированные слои исследуемых образцов выращивались при разных значениях $T_{g}$ от 350 до $510^{\circ} \mathrm{C}$, а значение $\gamma$ было одинаковым для всех образцов и равнялось 28. На рис. 1 представлено схематическое изображение поперечного

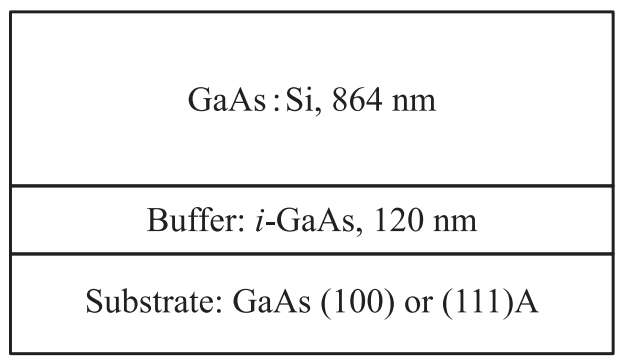

Рис. 1. Схематическое изображение исследуемых образцов в сечении.

сечения исследуемых образцов, а в табл. 1 приведены температуры роста легированного $\mathrm{Si}$ слоя $\mathrm{GaAs}$ для всех образцов.

Электрофизические характеристики (подвижности электронов и дырок, их концентрации) были определены с помощью измерений удельного сопротивления и эффекта Холла четырехзондовым методом в геометрии Ван дер Пау при комнатной температуре. При измерении спектров ФЛ образцы находились в комбинированном оптическом криостате в атмосфере азота при температуре $77 \mathrm{~K}$. Для возбуждения ФЛ использовалось сфокусированное излучение твердотельного лазера с длиной волны 405 нм и мощностью 230 мВт. Детектором сигнала в области $1.2-2.0$ эВ являлся охлаждаемый жидким азотом фотоэлектронный умножитель ФЭУ-79 (катод S1). Энергетическое разрешение спектров ФЛ составляло $\sim 5$ мэВ.

\section{3. Результаты исследований и их обсуждение}

\section{1. Электрофизические параметры}

В табл. 1 представлены значения подвижности $\mu$, тип и концентрации носителей заряда в легированных $\mathrm{Si}$ слоях GaAs, определенные при температуре $300 \mathrm{~K}$, а на рис. 2 представлены соответствующие зависимости параметров от температуры роста.

Как видно из табл. 1, все образцы, выращенные на подложках $\mathrm{GaAs}$ (100), имеют $n$-тип проводимости. С уменьшением температуры роста эпитаксиальной пленки концентрация электронов монотонно уменьшается от $3.8 \cdot 10^{18}$ до $1.8 \cdot 10^{16} \mathrm{~cm}^{-3}$, также уменьшается их подвижность от 1773 до $176 \mathrm{~cm}^{2} /(\mathrm{B} \cdot \mathrm{c})$. Наблюдается монотонное снижение эффективности донорного легирования $\mathrm{Si}$. Такое поведение концентрации электронов может объясняться тем, что из-за амфотерности кремния атомы $\mathrm{Si}$ в кристаллической решетке $\mathrm{GaAs}$ занимают узлы $\mathrm{Ga}\left(\mathrm{Si}_{\mathrm{Ga}}\right)$, узлы $\mathrm{As}\left(\mathrm{Si}_{\mathrm{As}}\right)$ и межузельные положения. В зависимости от условий роста соотношение количеств $\mathrm{Si}_{\mathrm{Ga}}$ и $\mathrm{Si}_{\mathrm{As}}$ может различаться и, как следствие, будет различаться концентрация носителей заряда. В случае образцов на подложках GaAs (100) при уменьшении 
Таблица 1. Температура роста слоя $\mathrm{GaAs}: \mathrm{Si}\left(T_{g}\right)$ и электрофизические параметры образцов

\begin{tabular}{|c|c|c|c|c|c|}
\hline $\begin{array}{l}\text { Номер } \\
\text { образца }\end{array}$ & $T_{g},{ }^{\circ} \mathrm{C}$ & $\begin{array}{c}\text { Ориентация } \\
\text { подложки }\end{array}$ & $\begin{array}{c}\text { Тип } \\
\text { носителей } \\
\text { заряда }\end{array}$ & $\begin{array}{c}\text { Подвижность, } \\
\mathrm{cm}^{2} /(\mathrm{B} \cdot \mathrm{c})\end{array}$ & $\begin{array}{c}\text { Концентрация, } \\
\mathrm{cm}^{-3}\end{array}$ \\
\hline 997 & 510 & $\begin{array}{c}(100) \\
(111) \mathrm{A}\end{array}$ & $\begin{array}{c}\text { Электроны } \\
\text { Дырки }\end{array}$ & $\begin{array}{r}1773 \\
94\end{array}$ & $\begin{array}{l}3.8 \cdot 10^{18} \\
3.3 \cdot 10^{18}\end{array}$ \\
\hline 998 & 470 & $\begin{array}{c}(100) \\
(111) \mathrm{A}\end{array}$ & $\begin{array}{c}\text { Электроны } \\
\text { Дырки }\end{array}$ & $\begin{array}{r}1743 \\
97\end{array}$ & $\begin{array}{l}3.18 \cdot 10^{18} \\
2.46 \cdot 10^{18}\end{array}$ \\
\hline 999 & 430 & $\begin{array}{c}(100) \\
(111) \mathrm{A}\end{array}$ & $\begin{array}{c}\text { Электроны } \\
\text { Дырки }\end{array}$ & $\begin{array}{r}485 \\
33\end{array}$ & $\begin{array}{l}4.4 \cdot 10^{17} \\
1.1 \cdot 10^{18}\end{array}$ \\
\hline $23 \mathrm{~V}$ & 410 & $\begin{array}{c}(100) \\
(111) \mathrm{A}\end{array}$ & $\begin{array}{c}\text { Электроны } \\
\text { Дырки }\end{array}$ & $\begin{array}{r}444 \\
54\end{array}$ & $\begin{array}{l}2.3 \cdot 10^{17} \\
4.4 \cdot 10^{13}\end{array}$ \\
\hline 1000 & 380 & $\begin{array}{c}(100) \\
(111) \mathrm{A}\end{array}$ & $\begin{array}{c}\text { Электроны } \\
-\end{array}$ & $\begin{array}{l}121 \\
-\end{array}$ & $\begin{array}{c}0.9 \cdot 10^{16} \\
-\end{array}$ \\
\hline $25 \mathrm{~V}$ & 350 & $\begin{array}{l}(100) \\
(111) \mathrm{A}\end{array}$ & $\begin{array}{c}\text { Электроны } \\
\text { Дырки }\end{array}$ & $\begin{array}{r}176 \\
68\end{array}$ & $\begin{array}{l}1.8 \cdot 10^{16} \\
5.8 \cdot 10^{13}\end{array}$ \\
\hline
\end{tabular}
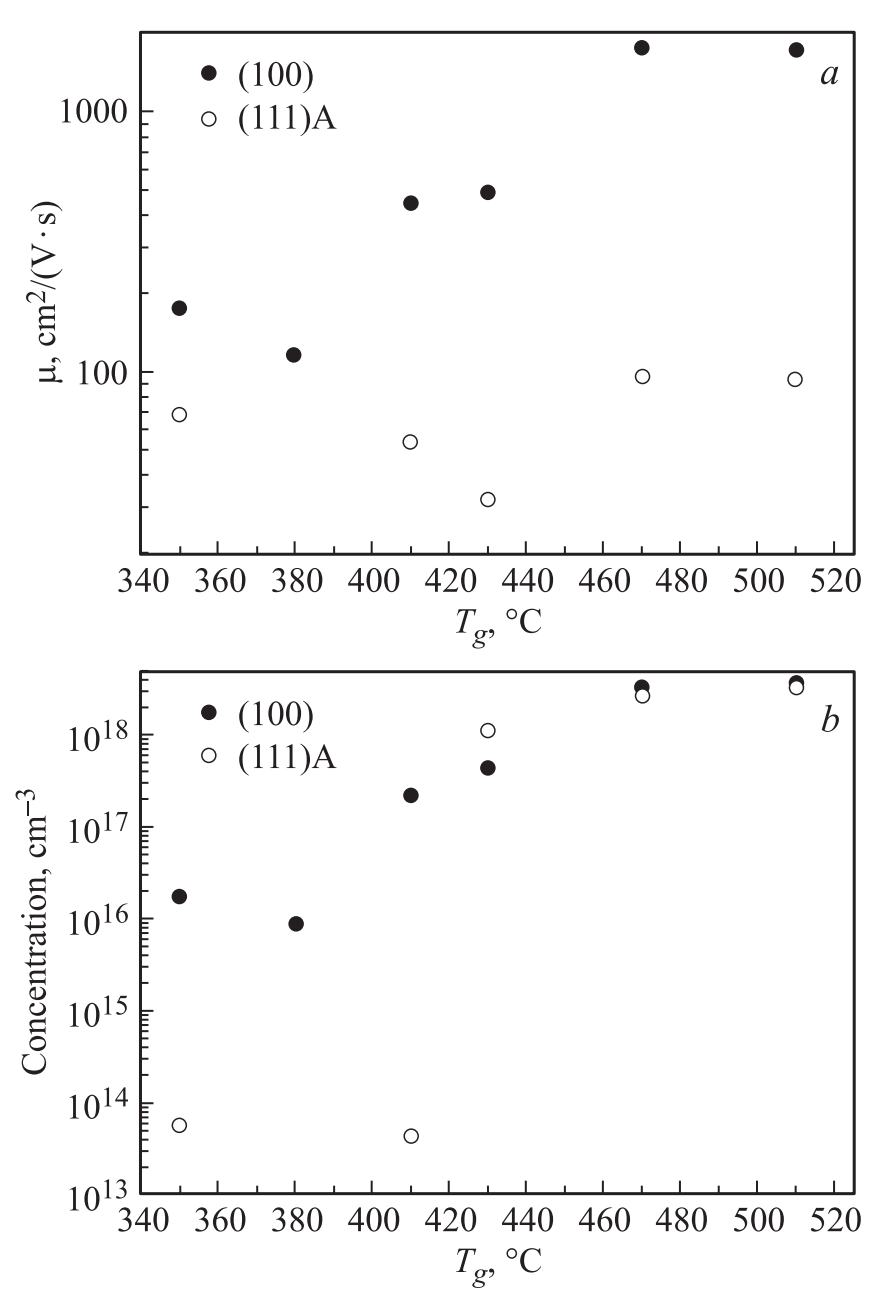

Рис. 2. Зависимости подвижности $(a)$ и концентрации $(b)$ носителей заряда от температуры роста образцов. температуры роста количество атомов $\mathrm{Si}$ в позициях $\mathrm{Si}_{\mathrm{Ga}}$ уменьшается с одновременным увеличением концентрации $\mathrm{Si}_{\mathrm{As}}$, что приводит к снижению концентрации электронов. Другой причиной уменьшения концентрации электронов может быть образование в слоях GaAs при понижении температуры роста компенсирующих доноры $\mathrm{Si}$ точечных дефектов, таких как $V_{\mathrm{Ga}}$, что будет обсуждаться далее в разделе по фотолюминесценции.

Как видно из табл. 1, наблюдается снижение подвижности электронов с понижением температуры роста. Концентрация внедренных атомов $\mathrm{Si}$ в нашем случае постоянна для всех образцов. Поэтому, на наш взгляд, снижение подвижности связано с увеличением концентрации не связанных с Si структурных дефектов, приводящих к усилению рассеяния электронов.

Из данных табл. 1 следует, что эпитаксиальные пленки GaAs на подложках GaAs (111)A имеют $p$-тип проводимости при $\gamma=28$ в диапазоне $T_{g}$ от 430 до $510^{\circ} \mathrm{C}$. При $T_{g}<410^{\circ} \mathrm{C}$ эпитаксиальные пленки становятся настолько высокоомными, что их проводимость оказывается сравнимой с проводимостью полуизолирующей подложки, и достоверно измерить электрофизические параметры не удается. Возникновение $p$-типа проводимости в легированных $\mathrm{Si}$ пленках $\mathrm{GaAs}$ с ориентацией (111)A обусловлено ростом в условиях недостатка мышьяка и объясняется низким коэффициентом прилипания атомов As к поверхности (111)A при стандартных температурах роста [20]. Это способствует преимущественному встраиванию легирующих атомов $\mathrm{Si}$ в узлы As. При снижении температуры роста уменьшается вероятность десорбции атомов и молекул мышьяка с ростовой поверхности, что должно приводить к снижению доли $\mathrm{Si}_{\mathrm{As}}$ и уменьшению концентрации дырок. В отличие от образцов на подложках GaAs (100), для подложек (111)A 
концентрация свободных носителей не постепенно снижается во всем диапазоне исследованных температур роста, а скачкообразно уменьшается при температурах роста $\lesssim 410^{\circ} \mathrm{C}$. Можно предположить, что существует минимальная критическая температура роста для получения $p$-типа проводимости пленок $\mathrm{GaAs}: \mathrm{Si}$ (111)A, которая зависит от $\gamma$. В нашем случае, для $\gamma=28$ эта критическая температура находится в диапазоне $T_{g}=410-430^{\circ} \mathrm{C}$.

\section{2. Спектроскопия фотолюминесценции}

Природа особенностей спектров ФЛ GaAs в диапазоне энергий фотонов 1.20-1.52 эВ обсуждается в работах [15,21-26]. В области примесной ФЛ (т.е. в диапазоне $1.20-1.48$ эВ) особенности в спектрах ФЛ связывают с дефектами типа $\mathrm{As}_{\mathrm{Ga}}, V_{\mathrm{Ga}}$ и комплексами дефектов $\mathrm{As}_{\mathrm{Ga}}-\mathrm{Si}_{\mathrm{Ga}}, V_{\mathrm{Ga}}-\mathrm{Si}_{\mathrm{As}}$. Разделение вклада разных дефектов в излучение в указанном диапазоне усложняется тем, что упомянутые дефекты могут быть как в нейтральном $\left(\mathrm{As}_{\mathrm{Ga}}^{0}, V_{\mathrm{Ga}}^{0}\right)$, так и в заряженных состояниях $\left(\mathrm{As}_{\mathrm{Ga}}^{+}, \mathrm{As}_{\mathrm{Ga}}^{+2}, V_{\mathrm{Ga}}^{-}, V_{\mathrm{Ga}}^{-2}, V_{\mathrm{Ga}}^{-3}\right)$ [23]. Кроме того, исследуемые нами образцы представляют собой легированные кремнием структуры на подложках $\mathrm{GaAs}$ с ориентациями (100) и (111)А. Амфотерные свойства кремния как легирующей примеси в эпитаксиальных слоях GaAs, выращенных на таких подложках, проявляются по-разному, и поэтому мы получаем разный тип и разную концентрацию носителей заряда при одинаковой концентрации внедренных атомов Si. Поскольку легирование по-разному влияет на концентрацию заряженных атомов примеси, полная концентрация дефектов $\mathrm{As}_{\mathrm{Ga}}$ и $V_{\mathrm{Ga}}$ может изменяться из-за уровня легирования (так называемый эффект уровня Ферми [23]), а также из-за различных условий эпитаксиального роста.

На рис. 3 представлены спектры ФЛ образцов 997, 998, 999 и 23V, выращенных на подложках GaAs c ориентациями (100) и (111)A при $T_{g} \geq 410^{\circ} \mathrm{C}$. Пары пиков в спектрах ФЛ образцов 999-0 и 23V-1 вблизи 1.5 эВ, отмеченные стрелками (laser), соответствуют не полностью отфильтрованному излучению лазера во втором порядке дифракции монохроматора.

Рассмотрим спектры ФЛ образцов, полученных на подложках GaAs (100). Как видно из рис. 3, экспериментальный спектр ФЛ образца 997-0 хорошо описывается суммой трех гауссианов: краевой полосы с энергией максимума $\hbar \omega_{0}=1.562$ эВ и двух полос излучения в примесной области спектра с энергиями максимумов $\hbar \omega_{1}=1.440$ эВ и $\hbar \omega_{2}=1.363$ эВ. Экспериментальный спектр ФЛ образца 998-0 также описывается суммой трех гауссианов с энергиями максимумов $\hbar \omega_{0}=1.558$ эВ, $\hbar \omega_{1}=1.436$ эВ и $\hbar \omega_{2}=1.343$ эВ. Максимум сигнала краевой ФЛ образцов 977-0 и 978-0 (пик 0 на рис. 3) смещен в сторону бо́льших энергий относительно ширины запрещенной зоны GaAs за счет вырождения электронов (эффект Бурштейна-Мосса). При этом пик межзонной люминесценции образца 997-0 смещен в сторону бо́льших энергий по сравнению с образцом 998-0, в соответствии с измерениями концентрации электронов.

В примесной области, т.е. в диапазоне энергий фотонов 1.20-1.48 эВ, согласно литературным данным, в спектрах ФЛ пленок $\mathrm{GaAs}$ : $\mathrm{Si}$ наблюдаются особенности при $\hbar \omega_{2} \approx 1.35$ эВ и $\hbar \omega_{1} \approx 1.45$ эВ. Если первая из них обычно приписывается комплексу $\mathrm{Si}_{\mathrm{As}}-V_{\mathrm{As}}$, то данные о природе второй особенности противоречивы [24]. Наиболее вероятным считается, что полоса ФЛ с энергией $\hbar \omega_{1} \approx 1.45$ эВ связана с рекомбинацией электронов и дырок на глубоких акцепторных уровнях, которые приписываются нейтральным дефектам $V_{\mathrm{Ga}}$ и $\mathrm{Ga}_{\mathrm{As}}$ [25].

Как следует из анализа примесных полос в экспериментально измеренных спектрах ФЛ, отношение интенсивностей $I_{1} / I_{0}$ полос с $\hbar \omega_{1}$ и $\hbar \omega_{0}$ для обоих образцов, 997-0 и 998-0, примерно одинаковое (2.3 и 2.1 соответственно), а отношение интенсивностей $I_{2} / I_{0}$ полос с $\hbar \omega_{2}$ и $\hbar \omega_{0}$ для образца 997-0 больше, чем для образца 998-0 (1.2 и 0.6 соответственно). Такое поведение полосы $\hbar \omega_{2}$ мы связываем с тем, что образец 997-0 был выращен при более высокой температуре. В этом случае количество уходящих атомов мышьяка с поверхности образца увеличивается и поэтому более вероятно увеличение концентрации $V_{\mathrm{As}}$ и образование комплексов $\mathrm{Si}_{\mathrm{As}}-V_{\mathrm{As}}$. При таком предположении поведение полосы $\hbar \omega_{2}$ связано с тем, что концентрация $V_{\mathrm{As}}$ превышает концентрацию $\mathrm{Si}_{\mathrm{As}}-V_{\mathrm{As}}$.

При снижении температуры роста LT-GaAs : Si (100) до $430-410^{\circ} \mathrm{C}$ (образцы 999 и $\left.23 \mathrm{~V}\right)$ происходит уменьшение интенсивности сигнала ФЛ и исчезает возможность четко выделить различные области спектра. Видно, что сигнал ФЛ регистрируется в диапазоне энергий от 1.5-1.6 до 1.2 эВ. Наблюдается появление доминирующего пика ФЛ с энергией максимума $\hbar \omega<1.2$ эВ, находящегося за границей чувствительности фотоприемника. Можно предположить, что эта широкая полоса соответствует излучательной рекомбинации с участием дефектов $\mathrm{Si}_{\mathrm{Ga}}-V_{\mathrm{Ga}}$. Их образованию в нашем случае могло способствовать снижение температуры роста (эквивалентное повышению потока атомов As). Согласно литературным данным, в условиях избытка атомов As дефекты $\mathrm{Si}_{\mathrm{Ga}}-V_{\mathrm{Ga}}$ создают широкую и интенсивную полосу ФЛ в диапазоне $0.9-1.3$ эВ с максимумом при $1.1-1.2$ эВ $[24,25]$.

При дальнейшем снижении температуры роста $\left(T_{g} \leq 380^{\circ} \mathrm{C}\right)$ в спектрах ФЛ низкотемпературных пленок 1000 и $25 \mathrm{~V}$ (не приведены) остается только интенсивный пик ФЛ в диапазоне $\hbar \omega<1.35$ эВ с максимумом за границей чувствительности приемника. Таким образом, при последовательном снижении температуры роста при фиксированном $\gamma$ в спектрах ФЛ пленок $\mathrm{GaAs}: \mathrm{Si}$ (100) ослабевает интенсивность оптических переходов, связанных с дефектами $V_{\mathrm{As}}$ и атомами $\mathrm{Si}_{\mathrm{As}}$, и увеличивается интенсивность переходов, связанных c $V_{\mathrm{Ga}}$. Это указывает на соответствующие изменения 

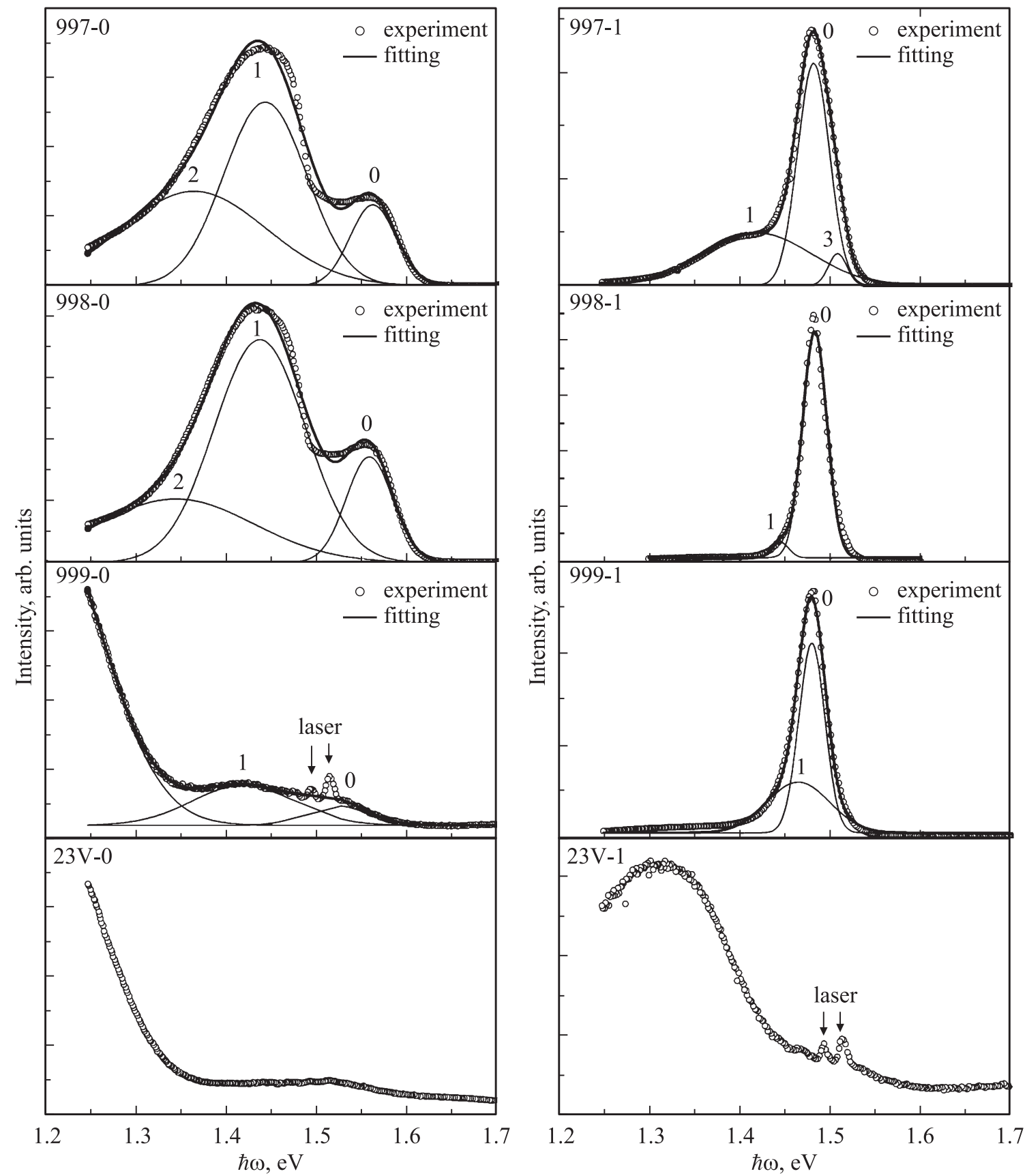

Pис. 3. Спектры ФЛ пленок LT-GaAs : Si на подложках (100) и (111)A, полученных при температурах $T_{g} \geq 410^{\circ} \mathrm{C} .0,1,2,3-$ гауссианы (см. табл. 2).

концентрации этих дефектов с $T_{g}$. Именно с увеличением концентрации $V_{\mathrm{Ga}}$, являющихся акцепторами, при уменьшении $T_{g}$ мы связываем наблюдаемое монотонное уменьшение концентрации свободных электронов в пленках LT-GaAs : Si (100).

Рассмотрим спектры ФЛ образцов, полученных на подложках GaAs (111)A. Результаты разложения спектров ФЛ образцов 997-1, 998-1 и 999-1на гауссианы приведены в табл. 2. Согласно электрофизическим измерениям образец 997-1 обладает самой большой концентрацией дырок (табл. 2). Спектр образца 997-1 выглядит как типичный спектр ФЛ вырожденного GaAs p-типа проводимости [26]. Наблюдаются три основные полосы с энергиями $\hbar \omega_{0}=1.507,1.48$ эВ и $\hbar \omega_{1}=1.420$ эВ. Полоса с $\hbar \omega_{0}=1.507$ эВ соответствует межзонной электроннодырочной рекомбинации в вырожденном $p$-GaAs. Полоса с $\hbar \omega_{0}=1.48$ эВ, наблюдавшаяся для GaAs c $p$-типом проводимости (концентрации $\geq 10^{18} \mathrm{~cm}^{-3}$ ) на подложках GaAs (111)A в работе [26], связывается с переходами между зоной проводимости и акцепторами $\mathrm{Si}_{\mathrm{As}}$, а также между центрами $\mathrm{Si}_{\mathrm{As}}-\mathrm{Si}_{\mathrm{Ga}}$, что вызвано высокими концентрациями донорных и акцепторных уровней. 
Таблица 2. Параметры гауссианов $0,1,2,(3)$ разложения спектров (рис. 3) ФЛ исследуемых образцов: энергетическое положение $\hbar \omega$, ширина на половине высоты FWHM, высота, нормированная на высоту наибольшего из гауссианов в спектре ФЛ, $I_{\text {norm }}$

\begin{tabular}{c|c|c|c|c|c|c|c|c|c}
\hline \multirow{2}{*}{ Образец } & \multicolumn{3}{|c|}{2} & \multicolumn{3}{|c|}{1} & \multicolumn{3}{c}{0} \\
\cline { 2 - 9 } & $\hbar \omega$, эB & FWHМ, эB & $I_{\text {norm }}$ & $\hbar \omega$, эB & FWHM, эB & $I_{\text {norm }}$ & $\hbar \omega$, эB & FWHM, эB & $I_{\text {norm }}$ \\
\hline $997-0$ & 1.363 & 0.160 & 0.51 & 1.442 & 0.092 & 1.00 & 1.562 & 0.050 \\
$998-0$ & 1.343 & 0.173 & 0.29 & 1.436 & 0.101 & 1.00 & 1.558 & 0.051 \\
$999-0$ & - & - & - & 1.418 & 0.133 & 1.00 & 1.522 & 0.081 \\
$997-1$ & $1.507^{*}$ & $0.022^{*}$ & $0.17^{*}$ & 1.424 & 0.110 & 0.23 & 1.480 & 0.034 \\
$998-1$ & - & - & - & 1.444 & 0.018 & 0.07 & 1.482 & 0.027 & 1.00 \\
$999-1$ & - & - & - & 1.461 & 0.068 & 0.27 & 1.476 & 0.028 & 1.00 \\
& & & & & & & & &
\end{tabular}

Примечание. ${ }^{*}$ относится к гауссиану 3 на рис. 3 .

Присутствует широкая полоса с энергией $\hbar \omega_{1}=1.42$ эВ, которую обычно приписывают заряженным дефектам $V_{\mathrm{As}}^{+}$. Энергетическое положение пика, связанного с такими дефектами, может меняться в зависимости от мощности возбуждения [13]. Отсутствие данной полосы в спектрах ФЛ образцов 998-1 и 999-1, по-видимому, связано с уменьшением концентрации дефектов $V_{\text {As. }}$ Поскольку температура роста этих образцов ниже, чем образца 997-1, реиспарение атомов As во время роста уменьшается, а это приводит к уменьшению концентрации $V_{\text {As }}$.

При температурах роста $T_{g} \lesssim 410^{\circ} \mathrm{C}$ пленок $\mathrm{GaAs}: \mathrm{Si}$ (111)A в спектрах ФЛ появляется и становится доминирующей полоса с энергией $\hbar \omega<1.35$ эВ с максимумом при 1.2-1.3 эВ, соответствующая переходам с участием $V_{\mathrm{Ga}}$ и комплексов $\mathrm{Si}_{\mathrm{Ga}}-V_{\mathrm{Ga}}$. Отметим, что переходы с участием этих центров для пленок $\mathrm{GaAs}: \mathrm{Si}$, выращенных на подложках (111)A, появляются при более низкой температуре роста $\left(410^{\circ} \mathrm{C}\right.$, образец $\left.23 \mathrm{~V}-1\right)$, чем для подложек (100) $\left(430^{\circ} \mathrm{C}\right.$, образец 999).

Одновременно с появлением в спектрах ФЛ полосы $V_{\mathrm{Ga}}$ наблюдается скачкообразное снижение концентрации свободных дырок в $\mathrm{GaAs}: \mathrm{Si}$ на подложках (111)A. По-видимому, избыток мышьяка при низких температурах роста, приводящий к формированию вакансий галлия, уменьшает вероятность формирования акцепторных центров $\mathrm{Si}_{\mathrm{As}}$ при $T_{g} \lesssim 410^{\circ} \mathrm{C}$.

На рис. 4 представлены зависимости интегральной интенсивности ФЛ образцов в диапазоне энергий фотонов 1.2-1.8 эВ от температуры роста. Как видно из рис. 4, интегральная интенсивность ФЛ уменьшается по мере уменьшения $T_{g}$. Для образцов, выращенных при высоких температурах $\left(430-510^{\circ} \mathrm{C}\right)$, отметим следующую закономерность: образцы, выращенные на подложках GaAs (111)A, люминесцируют ярче, чем образцы на подложках $\mathrm{GaAs}$ (100). При низких температурах роста $\left(350-410^{\circ} \mathrm{C}\right)$ наблюдается обратная ситуация: теперь ярче люминесцируют образцы, выращенные на подложках GaAs (100). При температурах роста 430 и $410^{\circ} \mathrm{C}$ (вблизи критической температуры получения $p$-типа проводимости на подложках GaAs (111)A) для образцов

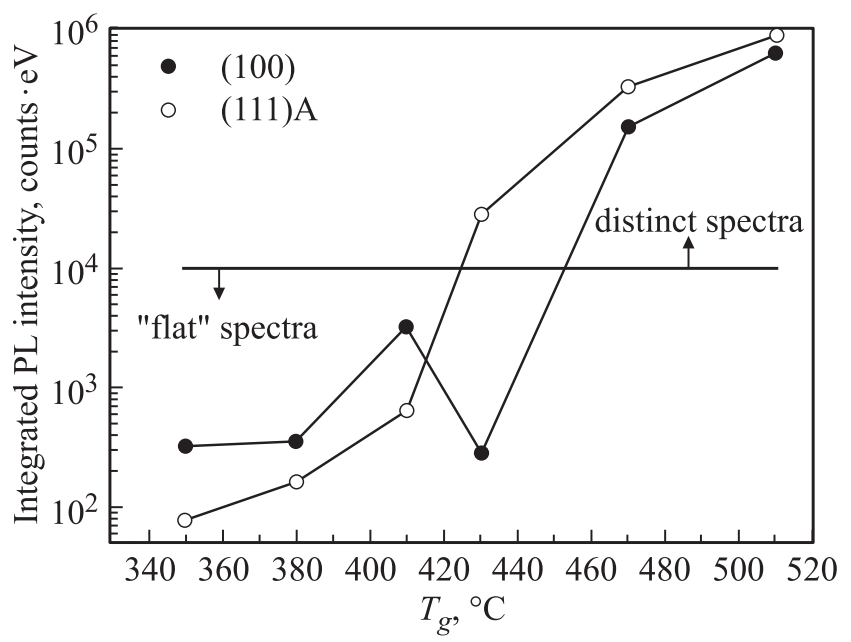

Рис. 4. Зависимости интегральной интенсивности фотолюминесценции (PL) от температуры роста образцов.

на подложках GaAs (100) и (111)A соответственно кроме резкого уменьшения интегральной интенсивности ФЛ происходит кардинальное изменение формы спектра ФЛ: исчезают заметные пики, и спектр становится относительно плоским. Это вызвано увеличением роли безызлучательной рекомбинации фотовозбужденных электронов и дырок из-за возрастания концентрации точечных дефектов при понижении температуры роста.

\section{4. Заключение}

В работе впервые проведены исследования электрофизических параметров и спектров фотолюминесценции однородно легированных атомами $\mathrm{Si}$ пленок GaAs, выращенных методом молекулярно-лучевой эпитаксии на подложках GaAs с кристаллографической ориентацией поверхности (111)А при пониженных температурах роста. Результаты исследований представлены в сравнении с результатами для таких же пленок на подложках GaAs с традиционной ориентацией (100). Для идентичности технологических условий роста образцов в каждом про- 
цессе роста при выбранной температуре одновременно выращивались образцы на подложках GaAs (100) и (111)A. Давление $\mathrm{As}_{4}$ выбиралось из соображения получения $p$-типа проводимости на GaAs (111)A при стандартных температурах роста.

Выявлено, что при $\gamma \approx 28$ эпитаксиальные пленки $\mathrm{GaAs}: \mathrm{Si}$ на подложках $\mathrm{GaAs}$ (100) проявляют $n$-тип проводимости при понижении температуры роста от 510 вплоть до $350^{\circ} \mathrm{C}$. В случае использования подложек GaAs (111)A такие пленки проявляют p-тип проводимости при понижении температуры роста от 510 до $430^{\circ} \mathrm{C}$. Ниже $T_{g}=430^{\circ} \mathrm{C}$ пленки GaAs: $\mathrm{Si}(111) \mathrm{A}$ при $\gamma \approx 28$ являются полуизолирующими.

В спектрах ФЛ кроме основной полосы, характерной для вырожденного GaAs $n$ - и $p$-типа проводимости, отмечены примесные полосы в диапазоне энергий фотонов 1.25-1.46 эВ, которые мы можем отнести к дефектам $V_{\mathrm{Ga}}, V_{\mathrm{As}}, V_{\mathrm{As}}^{+}$и комплексам дефектов $\mathrm{Si}_{\mathrm{As}}-V_{\mathrm{As}}$.

Работа выполнена при поддержке РФФИ (грант № 16-29-03294 офи_м). Авторы выражают благодарность А.Н. Виниченко (НИЯУ МИФИ) за помощь в измерениях проводимости пленок.

\section{Список литературы}

[1] A. Krotkus. J. Phys. D: Appl. Phys., 43 (27), 273001 (2010).

[2] H. Eusebe, J.-F. Roux, J.-L. Coutaz, A. Krotkus. J. Appl. Phys., 98 (3), 033711 (2005).

[3] Г.Б. Галиев, Е.А. Климов, Д.В. Лаврухин, А.Э. Ячменев, Р.Р. Галиев, Д.С. Пономарев, Р.А. Хабибуллин, Ю.В. Федоров, А.С. Бугаев. Нано- и микросистемная техника, 6, $28(2014)$

[4] A. Krotkus, K. Bertulis, L. Dapkus, U. Olin, S. Marcinkevičius. Appl. Phys. Lett., 75 (21), 3336 (1999).

[5] J.-L. Coutaz, J.-F. Roux, A. Gaarder, S. Marcinkevičius, J. Jasinski, K. Korons, M. Kaminska, K. Bertulis, A. Krotkus. XI International Semiconducting and Insulating Materials Conf. (Canberra, Australia, 2000) p. 89.

[6] G. Segschneider, F. Jacob, T. Löffler, H.G. Roskos, S. Tautz, P. Kiesel, G. Döhler. Phys. Rev. B, 65, 125205 (2002).

[7] M.C. Beard, G.M. Turner, C.A. Schmuttenmaer. J. Appl. Phys., 90 (12), 5915 (2001).

[8] M. Haiml, U. Siegner, F. Morier-Genoud, U. Keller, M. Luysberg, P. Specht, E.R. Weber. Appl. Phys. Lett., 74 (9), 1269 (1999).

[9] P. Specht, S. Jeong, H. Sohn, M. Luysberg, A. Prasad, J. Gebauer, R. Krause-Rehberg, E.R. Weber. Mater. Sci. Forum, 258-263, 951 (1997).

[10] Г.Б. Галиев, В.Г. Мокеров, Ю.В. Слепнев, Ю.В. Хабаров, А.А. Ломов, Р.М. Имамов. ЖТФ, 69 (7), 68 (1999).

[11] G. Galiev, V. Kaminskii, D. Milovzorov, L. Velihovskii, V. Mokerov. Semicond. Sci. Technol., 17 (2), 120 (2002).

[12] L. Pavesi, F. Piazza, M. Henini, I. Harrison. Semicond. Sci. Technol., 8 (2), 167 (1993).

[13] F. Piazza, L. Pavesi, M. Henini, D. Johnston. Semicond. Sci. Technol., 7 (12), 1504 (1992).

[14] K. Agawa, K. Hirakawa, N. Sakamoto, Y. Hashimoto, T. Ikoma. Appl. Phys. Lett., 65 (9), 1171 (1994).
[15] Nguyen Hong Ky, F.K. Reinhart. J. Appl. Phys., 83 (2), 718 (1998).

[16] J.T. Schick, C.G. Morgan, P. Papoulias. Phys. Rev. B, 66 (19), 195302 (2002).

[17] D.J. Chadi, S.B. Zhang. Phys. Rev. B, 41 (8), 5444 (1990).

[18] M. Luysberg, H. Sohn, A. Prasad, P. Specht, Z. LilientalWeber, E.R. Weber, R. Krause-Rehberg. J. Appl. Phys., 83 (1), 561 (1998)

[19] D. Johnston, L. Pavesi, M. Henini. Microelectronics J., 26 (8), 759 (1995).

[20] M.R. Fahy, K. Sato, B.A. Joyce. Appl. Phys. Lett., 64 (2), 190 (1994).

[21] L. Pavesi, Nguyen Hong Ky, J.D. Caniere, F.K. Reinhart, N. Baba-Ali, I. Harrison, B. Tuck, M. Henini. J. Appl. Phys., 71, 2225 (1992).

[22] Г.Б. Галиев, Е.А. Климов, М.М. Грехов, С.С. Пушкарев, Д.В. Лаврухин, П.П. Мальцев. ФТП, 50 (2), 195 (2016).

[23] M.H. Zhang, Y.F. Zhang, Q. Huang, C.L. Bao, J.M. Sun, J.M. Zhou. J. Cryst. Growth, 209 (1), 37 (2000).

[24] И.А. Бобровникова, М.Д. Вилисова, И.В. Ивонин, Л.Г. Лаврентьева, В.В. Преображенский, М.А. Путято, Б.Р. Семягин, С.В. Субач, С.Е. Торопов. ФТП, 37 (9), 1072 (2003).

[25] L. Pavesi, M. Henini, D. Johnston. Appl. Phys. Lett., 66 (21), 2846 (1995).

[26] A. Miyagawa, T. Yamamoto, Y. Ohnishi, J.T. Nelson, T. Ohachi. J. Cryst. Growth, 237-239, 1434 (2002).

Редактор Л.В. Шаронова

\section{Photoluminescence investigation of silicon-doped GaAs films grown on (100) and (111)A GaAs substrates at reduced temperatures}

\section{G.B. Galiev, E.A. Klimov, A.N. Klochkov, S.S. Pushkarev, P.P. Maltsev}

Institute of Ultra High Frequency

Semiconductor Electronics,

Russian Academy of Sciences, 117105 Moscow, Russia

Abstract The electrophysical properties and photoluminescence spectra of the uniformly silicon-doped GaAs films obtained by molecular beam epitaxy on GaAs substrates with (100) and (111)A crystallographic orientations are investigated. The samples are grown at identical $\mathrm{As}_{4}$ pressures and at different growth temperatures varying from 350 to $510^{\circ} \mathrm{C}$. The (100)-grown samples demonstrate the $n$-type conductivity at all growth temperatures. The (111)A-grown samples demonstrate the $p$-type conductivity at growth temperatures $430-510^{\circ} \mathrm{C}$. The photoluminescence spectra of the samples contain edge interband and impurity luminescence bands. The edge band corresponds to the photoluminescence of the $n$ - or $p$-type degenerate GaAs. The impurity luminescence of the GaAs (100) samples at $1.3-1.45 \mathrm{eV}$ is attributed to the defects $V_{\mathrm{As}}$ and defect complexes $\mathrm{Si}_{\mathrm{As}}-V_{\mathrm{As}}$, the concentration of which depends on growth temperature. The transformation of the photoluminescence spectra with growth temperature of the (111)A GaAs samples is interpreted in terms of $V_{\mathrm{As}}$ and $V_{\mathrm{Ga}}$ defect concentration variations. 